\title{
Metal organic framework of rare earth tartrates.
}

\author{
Nazir Ahmad ${ }^{*}$, M .M. Ahmad ${ }^{1}$ and P. N. Kotru ${ }^{2}$ \\ ${ }^{1}$ Condensed Matter Physics Laboratory, Department of Physics, National Institute of Technology, \\ Hazratbal Srinagar - 190006, Jammu and Kashmir, India. \\ ${ }^{2}$ Crystal Growth and Materials Research Laboratory, Department of Physics and Electronics, \\ University of Jammu, Jammu-180006- India. \\ *Email: nazirnit@gmail.com
}

Single crystals of praseodymium tartrate dihydrate (PTD) possessing excellent ferroelectric, non-linear optical (NLO) properties and exhibiting remarkably flat habit faces, the most predominant being $\{101\}$, were successfully synthesized by gel technique for the first time. The smooth and flat habit faces depict that the grown crystals are free from defects and the same is supported by HRXRD studies. Microelectronic industry are now a days in search to find low-value dielectric materials which can serve the industry as inter-level dielectrics to offset the increase in signal propagation time between transistors, known as RC delay (where, $\mathrm{R}$ is metal wire resistance and $\mathrm{C}$ is inter-level dielectric capacitance).

Due to the use of low value dielectric material, the gate leakage current and heat dissipation can be brought down. Recently L- arginine oxalate, L-arginine acetate and praseodymium malonate were also reported to be low value dielectric materials $[1,2]$. Similar dielectric behavior has been observed in the grown crystal (PTD) as depicted in Figure 1.

The dielectric behavior recorded on $\{101\}$ planes of single crystals exhibit dielectric anomaly at $245{ }^{\circ} \mathrm{C}$, revealing a ferroelectric transition is supported by thermal and polarization versus electric field studies. The optical measurement helps to calculate the band gap $(5.13 \mathrm{eV})$ and is in good agreement with the studies of HRXRD and NLO behavior of the material. Absence of grain boundaries, thermal stability, ferroelectric and NLO behavior supports the grown single crystal to find its place in microelectronic industry as a multifunctional material.

\section{References}

1. M. Meena, C.K. Mahadevan. Mater.Lett.; 62 (2008) 3742-3744.

1. Nazir Ahmad, M. M. Ahmad, P. N. Kotru, RSC Advances 5 (2015) 93808-17

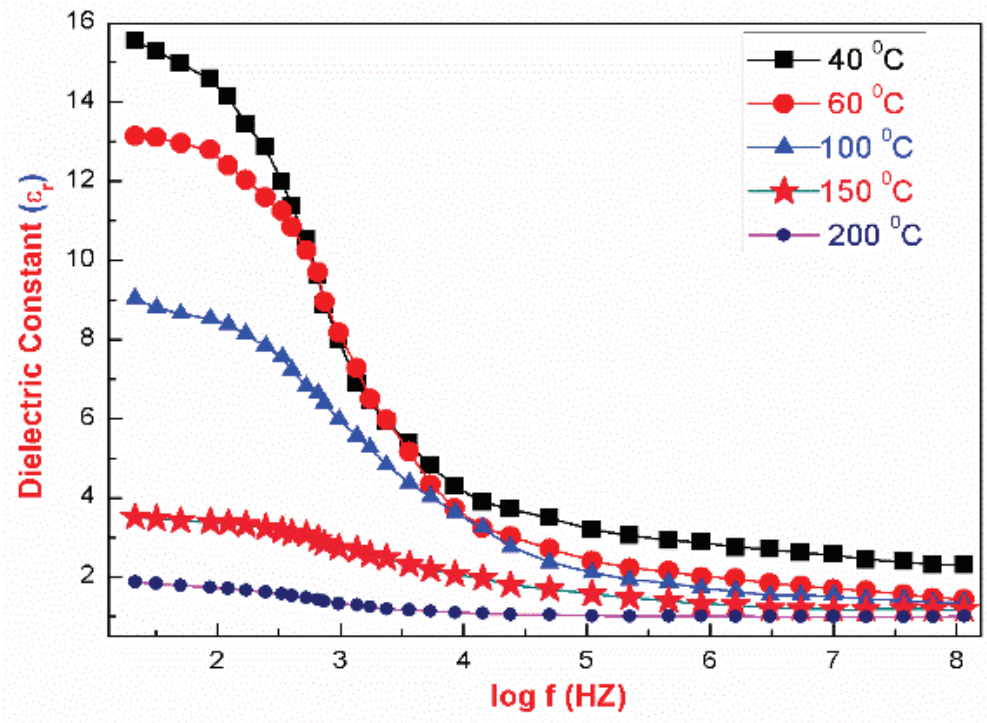

Figure 1: Dielectric constant verses frequency at different temperatures 